\title{
Application of Turbulent Mixing Noise Theory to Flows over Coanda Surfaces
}

\author{
Caroline Lubert \\ Department of Mathematics and Statistics, MSC 1911, James Madison University, Harrisonburg, VA 22807, USA
}

(Received 3 March 2007; revised 7 December 2007; accepted 22 February 2008)

\begin{abstract}
A wide variety of aeronautical and aerospace applications utilise the Coanda effect, or aspire to, due to the enhanced turbulence levels and entrainment that devices employing this effect generally offer when compared with conventional jet flows. However, such advantages are not usually achieved without a substantial increase in the corresponding acoustic radiation. This obviously detrimental side-effect has meant that in many cases the potential benefits of the Coanda effect have yet to be fully realised. Turbulent Mixing Noise (TMN) is a primary highfrequency noise source when the Coanda effect is employed. A theory has previously been developed to predict the TMN emitted by unit volume of jet-type shear-layer turbulence close to a rigid plane, and extended to a plane two-dimensional wall-jet. However, most flows of practical interest are three-dimensional, and often the surface is curved. This paper extends the previous models to predict the aeroacoustic characteristics of a threedimensional turbulent flow over a particular Coanda surface. Comparisons are made with experimental data, and comments are made regarding the generalisation of this theory to other Coanda surfaces.
\end{abstract}

\section{Nomenclature}

a - radius of a horizontal slice of jet

$a_{0} \quad-$ speed of sound

$b \quad-$ jet half-width

C - convection factor

$D_{n} \quad-$ slot width

$h-$ distance of observer plane vertically below origin

$k_{0}-$ constant (1.586)

$k \quad$ - wavenumber

$L_{x} \quad$ - longitudinal integral length scale

$\hat{n}-$ normal to the surface at the point of reflection

$p_{0} \quad-$ stagnation pressure

$p \quad-$ position vector of any point on flare-tip surface

$R \quad$ - distance of image source from point of reflection

$R_{c} \quad$ - radius of the flare centre stem

$R_{f} \quad-$ radius of the spherical section of the flare

$u^{\prime 2}-$ typical mean-square turbulence velocity

$\left(\overline{u^{\prime} v^{\prime}}\right)$ - maximum shear stress

$U_{m} \quad$ maximum mean velocity on the jet axis

$U_{j x} \quad-$ jet exit velocity

$\left(x_{1}, y_{1}, z_{1}\right)$ - point of reflection

$\left(x_{2}, y_{2}, z_{2}\right)$ - source position

$x_{n} \quad$ - distance along jet axis from nozzle

$y_{u} \quad-$ distance of source below surface (in $z=0$ plane)

$z_{S} \quad-$ perpendicular distance from source to the tangent plane that passes through the point of reflection of the source

$a$

- angle subtended by a radial line joining a sphere's centre to a point on the lower sphere surface and a vertical radial line

$a_{P C} \quad-$ angle $\alpha$ for a point at the end of the potential core

$\beta-$ angle of inclination of observer to downward vertical

$\delta-$ minimum distance from source to flare-tip surface

$\gamma \quad-$ angle between the observer direction and the source direction, in the $x-z$ plane
$\gamma_{L} \quad-$ limiting angle between the observer direction and the source direction, in the $x-z$ plane

$\rho_{0} \quad-$ ambient density

$\theta \quad-$ angle of reflection between observer and $\hat{n}$

$\omega_{f}-$ characteristic frequency emitted by the particular volume element of jet

\section{INTRODUCTION}

Turbulent Coanda wall-jets have become increasingly widely used in a variety of industrial applications in recent years, including circulation control wings, fan shrouds, thrust vectoring and combustors. ${ }^{1-15}$ Devices employing the Coanda effect usually offer substantial flow deflection, and enhanced turbulence levels and entrainment compared with conventional jet flows. However, these prospective advantages are generally accompanied by a significant increase in the associated noise levels. Consequently, in many cases, the full potential offered by the Coanda effect is yet to be completely realised. The development of a model for predicting the noise emitted by three-dimensional flows over Coanda surfaces would allow investigation of the effect of various flow parameters on the associated acoustic emission, and indicate ways in which the noise could be reduced or attenuated.

A theory has previously been developed for estimating the Turbulent Mixing Noise emitted by unit volume of jettype shear-layer turbulence in close proximity to an infinite rigid plane. ${ }^{16-20}$ Lighthill's $^{21}$ theory was applied to unit volume of free field turbulence, and an extension of this theory, developed by Curle $^{22}$ and Powell ${ }^{23}$ was used to take into account the presence of the rigid plane through a "Method of Images" approach, in which the original quadrupole is replaced by a quadrupole system comprising the original quadrupole plus an 'image quadrupole' representing the image of the original in the plane rigid boundary. Extending this work, predictions were made of some of the basic aeroacoustic characteristics associated with a plane two-dimensional wall-jet. ${ }^{18}$ 